\title{
Management of Breast Cancer during the COVID-19 Pandemic
}

\author{
Bulent Citgez, (1) Banu Yigit, (1) Emir Capkinoglu, (1) Sitki Gurkan Yetkin
}

Department of General Surgery, Sisli Hamidiye Etfal Medical Practice and Research Center, University of Health Sciences, Istanbul, Turkey

\begin{abstract}
The novel coronavirus disease (COVID-19) arises from the virus SARS-CoV-2 which is similar to the original SARS virus. The most common symptoms of the COVID-19 infection are fever, coughing and shortness of breath. According to the current data, the primary mode of transmission for the COVID-19 virus is between people through respiratory droplets and contact routes. The virus may lead to worse respiratory complications, including pneumonia, especially in older patients and patients with pre-existing illnesses, such as cancer. Cancer patients are at a significantly higher risk of getting infected with COVID-19 since their immune system can be compromised and that reality has to do with both that they have cancer and that they are on therapy for their cancer. COVID-19 crisis has impacted every aspect of the practice, including outpatient, elective, wards, emergency care, conferences, teaching and research. We should make sure cancer patients on active treatment are treated appropriately. In this review, we tried to explain how to prevent the negative effects of the COVID-19 pandemic on the diagnosis and treatment of breast cancer patients. Keywords: Breast cancer; COVID-19; coronavirus; SARS-COV-2; surgery.

Please cite this article as "Citgez B, Yigit B, Capkinoglu E, Yetkin GS. Management of Breast Cancer during the COVID-19 Pandemic. Med Bull Sisli Etfal Hosp 2020;54(2):132-135".
\end{abstract}

$\mathrm{T}$ he novel coronavirus disease (COVID-19), which was first described as an enveloped, positive-sense, singlestranded RNA viruses similar to SARS-CoV in the Hubei province of China in December 2019, began to appear in most countries outside China later. The World Health Organization (WHO) described this disease as a pandemic on 11 March. On the same date, the Republic of Turkey Ministry of Health has announced the first case in Turkey. On May 18, the WHO announced that there were 4833 022 cases and 317215 deaths confirmed worldwide. On this date, 149435 confirmed cases, and 4140 deaths was reported in Turkey and the number of recovered patients was 109962. ${ }^{[1]}$

The fatality rate of COVID-19 has been estimated at approximately 2 to $3 \%$, but it varies dramatically for different age groups and accompanying comorbidities like cardiovascular disease, diabetes, chronic respiratory disease, hypertension. ${ }^{[2]}$ However, it is not possible to say precisely the mortality rate, as the number of infected people is unknown, and the mortality rates also change if the virus is mutated. Patients are potential carriers during the incubation period in which the virus is contagious, but the patient does not display any symptoms. People with COVID-19 have had a wide range of symptoms reported - ranging from mild symptoms to severe illness. When patients become symptomatic, they may show many non-specific symptoms, including fever, cough, shortness of breath, repeated shaking with chills, muscle pain, headache, sore throat, the new loss of taste or smell. Although research for an effective drug or vaccine is still ongoing, potential vaccines must pass

Address for correspondence: Bulent Citgez, MD. Saglik Bilimleri Universitesi, Sisli Hamidiye Etfal Tip Uygulama ve Arastirma Merkezi, Genel Cerrahi Anabilim Dali, Istanbul, Turkey

Phone: +90 5052376847 E-mail: bcitgez@yahoo.com

Submitted Date: May 21, 2020 Accepted Date: June 01, 2020 Available Online Date: June 10, 2020

${ }^{\circ}$ Copyright 2020 by The Medical Bulletin of Sisli Etfal Hospital - Available online at www.sislietfaltip.org

OPEN ACCESS This is an open access article under the CC BY-NC license (http://creativecommons.org/licenses/by-nc/4.0/). 
through the clinical trial stages because it is primarily necessary for safety even during a pandemic. We still have to go on social distancing, contact tracing, self-isolation, and other measures.

In this process, as in many other types of cancer, there are also challenges in the management of breast cancer treatment due to the limited use of resources and the working discipline of the healthcare staff according to the pandemic conditions. Most of the clinicians around the world use the American Society of Breast Surgeons (ASBrS), the National Accreditation Program for Breast Centers (NAPBC), the National Comprehensive Cancer Network (NCCN), the Commission on Cancer ( $\mathrm{CoC}$ ) of the American College of Surgeons, and the American College of Radiology (ACR) guidelines as a standard for clinical decision-making during the worldwide COVID-19 pandemic. ${ }^{[3]}$ Our aims in the treatment of patients with breast cancer while figuring the epidemic are optimal long-term clinical outcomes for patients with breast cancer; low risk of infection or exposure among patients and staff; protection of patients from treatment-related side effects, such as immunosuppression, preservation of viral resources within the healthcare system, as our hospitals are strained by the health crisis.

\section{Classifications}

Following extensive discussions, the consortium of organizations and their representatives divided groups of patients with breast cancer according to three priorities as follows: ${ }^{[4]}$

1. Priority A: Patients in this group are patients who need to be treated immediately and have a disease that may threaten their life or progressively disturbing symptoms if treatment is not performed.

2. Priority B: Patients in this group are patients who do not need immediate treatment, but patients whose treatment cannot be postponed until the end of the long-term pandemic. The priority $B$ is divided into subgroups as follows: B1 (higher priority), B2 (mid-level priority), B3 (lower priority).

3. Priority C: Patients in this group are patients whose treatment can be delayed until the pandemic is over and patients who will not experience a negative result as a result of this delay.

Approaches and treatment suggestions (outpatient visit, diagnostics and imaging of breast disease, surgical oncology, radiation oncology and medical oncology) have been classified by the European Society for Medical Oncology (ESMO) as high, medium and low priority. ${ }^{[5]}$

1. High-priority: Recommendations are applied to patients whose condition is either clinically unstable or who have life-threatening cancer burden and requires immediate hospital treatment.

2. Medium-priority: Recommendations are applied to patients for whom delaying care beyond six weeks. Risk of delays caused by the COVID-19 pandemic are assessed, there should not be a concern that they may negatively affect this situation.

3. Low-priority: Recommendations are applied to patients for whom services can be delayed until the end of the COVID-19 pandemic.

American College of Surgeons (ACS), evaluated health system and hospitals in three phases by the community adequacy of resources, health workers:

1. Phase 1: The number of COVID-19 patients in hospitals is low and resources are sufficient. Neoadjuvant patients finishing treatment, clinical stage $\mathrm{T} 2$ or N1 ER +/ PR +/HER2-tumors, triple-negative or HER2 + patients, discordant biopsies likely to be malignant, excision of malignant recurrence are the cases need to be done as soon as possible.

2. Phase 2: The number of COVID-19 patients has increased; hospitals have limited resources. Incision and drainage of breast abscess, evacuation of a hematoma, revision of an ischemic mastectomy flap, and revision of an autologous tissue flap are the cases that need to be made as soon as possible.

3. Phase 3: Hospitals are full of COVID-19 patients; hospital resources are stated to be insufficient.

Incision and drainage of breast abscess, evacuation of a hematoma, revision of an ischemic mastectomy flap, and revision of an autologous tissue flap are the cases that need to be made as soon as possible.

Neoadjuvant chemotherapy may be considered in appropriate cases in phase 2 and phase 3 conditions. If institutional resources are sufficient in phase 1 conditions, it may be preferable to proceed with surgery instead of subjecting a patient to an immunocompromised state with neoadjuvant chemotherapy. ${ }^{[6]}$

\section{Imaging Studies}

All imaging studies, including mammography, ultrasound, and MRI, should be postponed until the COVID-19 period ends because there is no evidence of delaying screening mammography for the proposed short time will affect mortality. ${ }^{[7]}$ Diagnostic imaging for an abnormal mammogram or for suspicious symptoms of breast cancer, biopsies for BIRADS 4 or 5 lesions, and breast MRI for the extent of disease evaluation or pre-chemotherapy assessment are still being performed in hospitals having sufficient resources because 
pandemic may be long-lasting and this may threaten patients' lives in the long run. ${ }^{[4]}$

\section{Surgical Procedures}

Although we specifically talk about breast cancer treatment management, we would like to draw attention to some invariable rules in all surgical operations. All patients are questioned for signs of COVID-19 at admission, and patients with fever and respiratory symptoms are referred to the relevant departments during admission. In COVID-19 patients, operations are delayed if not urgent. Before surgery, all resources like devices, healthcare workers, intensive care beds, should be recorded against the possibility of being used at any time.

In the operating rooms, only the minimum number of staff must be employed alternately. Periodic training is given to the operating room staff about COVID-19 symptoms and prevention methods by digital methods. Personal protective equipment, including N95/FFP2 mask, sterile surgical gowns, disposable sterile gloves, goggles/protective visors, disposable bone, alcohol-based hand antiseptic, is provided to the healthcare staff in the operating room. Special stretcher, operating room, and elevator are reserved for patients who are infected with COVID-19 to be operated. Necessary precautions are taken before the operation, patient transfer, during and after the operation.

WHO, China's National Health Commission (NHC), The United States' CDC reported an incubation period for COVID-19 between $2-10,10-14,2-14$ days, respectively. ${ }^{[8-10]}$ As a result, although the incubation period is reported on average between 2-14 days, there is still no exact interval. Thus, we should assume everyone could be a carrier. Twenty-four hours before elective surgeries, PCR (Polymerase Chain Reaction) tests should be performed; patients should be isolated and visitors should not be taken to their rooms. If the PCR test result is positive, the operation of the patient should be postponed unless it is urgent; COVID-19 patients should be treated by following appropriate protocols. However, if the test result is negative, the test should be renewed every week as long as the patient stays in the hospital after the operation and PCR test should be performed before discharge. ${ }^{[11]}$

\section{Treatment Approach}

Many guidelines have common points in approaching breast cancer treatment, but patients are recommended to be evaluated on a case-by-case basis. Unless a complication, such as an abscess, infection, requires urgent treatment, surgery should be postponed for at least three months due to atypia, prophylactic/risk-reducing surgery, and benign breast disease. ER expressing types of atypical hyperplasia and lobular carcinoma in situ can be treated with tamoxifen or aromatase inhibitors. Although the main treatment of DCIS is breast-conserving surgery with or without whole breast radiation therapy, or alternatively, mastectomy, treatment management has changed due to pandemics. In this pandemic period, it is important to specify the hormone receptor status in DCIS-compliant core biopsies taken in the treatment of DCIS. ER + DCIS patients should be treated with endocrine therapy primarily and they should be checked via telehealth periodically. ER-DCIS patients with low volume and low risk of invasive disease can be followed periodically without treatment. If ER-DCIS is high grade, large volume or palpable, reassessments of patients using telemedicine should be more frequent. In DCIS with microinvasion, the treatment approach, such as $E R+$ invasive cancers is shown in ER + DCIS patients, while patients with ER- are treated like ER- DCIS patients. ${ }^{[12]}$

Generally, patients with early-stage breast cancer undergo primary surgery (breast-conserving surgery or mastectomy) with or without radiotherapy. Following local treatment, adjuvant systemic therapy may be recommended based on primary tumor characteristics, such as tumor size, stage, lymph node involvement, ER/PR status, and expression of the HER2 receptor. However, in the pandemic period, some priorities have also changed in the treatment of invasive breast cancer. ER+ early breast cancer patients should have neoadjuvant endocrine therapy before surgery during the COVID-19 pandemic. The decision of selection between Tamoxifen and aromatase inhibitors depends on patients' premenopausal or postmenopausal situation and hormonal status. If the patient is not postmenopausal, sequential evaluation of hormonal status is recommended to consider an alternative endocrine agent. ${ }^{[12]}$

The rate of pathologic complete response $(p C R)$ is higher in HER2 overexpressing (HER2+) and triple-negative (TN) breast cancers, and less frequent in the more common hormone- receptor-positive cancers. As a result, TN and Her2 + breast cancer patients with $\mathrm{T} 2$ or larger tumor or LN involvement are candidates for neoadjuvant therapy, and there was no significant difference in the approach in these patients during the pandemic process. Patients with T1N0M0 disease also encouraged to have NAC before surgery during the COVID-19 pandemic. ${ }^{[12]}$

Patients who are having NAC are at increased risk for COVID-19 infection because of immune deficiency. For the most part, symptoms of COVID-19 are the same in cancer patients as the general population. Medications in breast cancer patients receiving NAC can suppress the development of a fever or other symptoms, and COVID-19 disease may remain asymptomatic until they develop sepsis. If the patient tests 
positive for COVID-19 while receiving cancer treatment, we need to stop treatment until the patient recovers. ${ }^{[13]}$

\section{Reconstruction}

Reconstructive surgery procedures, such as delayed breast reconstruction and planned secondary or revision breast reconstruction, are elective processes and should be delayed until the pandemic is over. Immediate reconstruction after lumpectomy or mastectomy should be made by risk balancing due to the possibility of complications, prolonging of the hospitalization period and the need for additional surgeries. Immediate autologous flap reconstruction for breast reconstruction is elective and should be delayed, but decisions regarding immediate tissue expander or direct implant reconstruction should be made according to the hospital's resources and patient characteristics. ${ }^{[14]}$

\section{Conclusion}

In this review, we have explained some changes in the approach to breast cancer above, but the tailored therapy must be our first goal individually, depending on the healthcare workers, the materials and devices to be used, intensive care unit bed capacity and other resources. Breast cancer treatment requires a multidisciplinary approach. Dynamic changing of local conditions may affect the priority levels and treatment options. Regional ministry of health departments must have all hospital capacity for outpatient care. Patient treatment decisions should be made by thinking both during and after the pandemic management of healthcare systems. Social distancing and precautionary measures are still in effect. More recently, regions with low rates of infection are planning to gradually reopen their economies and begin lifting some of these measures. Unfortunately, remaining at home may be difficult for patients among us with severe medical conditions that require urgent attention, but COVID-19 impacts everything, including how we manage breast cancer.

\section{Disclosures}

Peer-review: Externally peer-reviewed.

Conflict of Interest: None declared.

Authorship Contributions: Concept - B.Ç.; Design - B.Y.; Supervision - B.Ç.; Materials - E.Ç., S.G.Y.; Data collection \&/or processing - S.G.Y., E.Ç.; Analysis and/or interpretation - B.Y.; Literature search - E.Ç., S.G.Y.; Writing - B.Ç.; Critical review - B.Y.

\section{References}

1. Worldometer. COVID-19 Coronavirus Pandemic. Available at: https:// www.worldometers.info/coronavirus /. Accessed May 18, 2020.

2. Worldometer. COVID-19 Coronavirus Pandemic. Available at: https:// www.worldometers.info/coronavirus/. Accessed Feb 23, 2020.
3. American Society of Breast Surgeons. Recommendations for Prioritization, Treatment and Triage of Breast Cancer Patients During the COVID-19 Pandemic: Executive Summary. Available at: https://www.breastsurgeons.org/docs/news/The_COVID-19_ Pandemic_Breast_Cancer_Consortium_Recommendations_EXECUTIVE_SUMMARY.pdf. Accessed Jun 03, 2020.

4. The COVID-19 Pandemic Breast Cancer Consortium. Available at: https://www.nccn.org/covid-19/pdf/The_COVID-19_Pandemic_ Breast_Cancer_Consortium_Recommendations.pdf. Accessed Jun 03, 2020.

5. Esmo management and treatment adapted recommendations in the covid-19 era: breast cancer. Available at: https://www.esmo.org/ guidelines/cancer-patient-management-during-the-covid-19-pandemic/breast-cancer-in-the-covid-19-era. Accessed Jun 03, 2020.

6. COVID-19 Guidelines for Triage of Breast Cancer Patients. Available at: https://www.facs.org/covid-19/clinical-guidance/elective-case/breast-cancer. Accessed Jun 03, 2020.

7. American Society of Breast Surgeons. ASBrS and ACR Joint Statement on Breast Screening Exams During the COVID-19 Pandemic. Available at: https://www.breastsurgeons.org/docs/news/202003-26-ASBrS-ACR-Joint-Statement.pdf. Accessed Jun 03, 2020.

8. World Health Organization (WHO). Novel Coronavirus (2019nCoV) Situation Report-7. Available at: https://www.who.int/docs/ default-source/coronaviruse/situation-reports/20200127-sitrep7-2019--ncov.pdf?sfvrsn=98ef79f5_2. Accessed Jun 03, 2020.

9. Al Jazeera. China's National Health Commission news conference on coronavirus. Available at: https://www.aljazeera.com/ news/2020/01/chinas-national-health-commission-news-conference-coronavirus-200126105935024.html. Accessed Jun 03, 2020.

10. Centers for Disease Control and Prevention. Symptoms of Novel Coronavirus (2019-nCoV). Available at: https://www.cdc.gov/ coronavirus/2019-ncov/symptoms-testing/symptoms.html. Accessed Jun 03, 2020.

11. Al-Muharraqi MA. Testing recommendation for COVID-19 (SARSCoV-2) in patients planned for surgery - continuing the service and 'suppressing' the pandemic. Br J Oral Maxillofac Surg 2020 Apr 13[Epub ahead of print], doi: 10.1016/j.bjoms.2020.04.014.

12. Resource for Management Options of Breast Cancer During COVID-19. Available at: https://www.surgonc.org/wp-content/uploads/2020/03/Breast-Resource-during-COVID-19-3.23.20.pdf. Accessed Jun 03, 2020.

13. Al-Shamsi HO, Alhazzani W, Alhuraiji A, Coomes EA, Chemaly RF, Almuhanna $M$, et al. A Practical Approach to the Management of Cancer Patients During the Novel Coronavirus Disease 2019 (COVID-19) Pandemic: An International Collaborative Group. Oncologist 2020 Apr 3 [Epub ahead of print], doi: 10.1634/theoncologist.2020-0213. [CrossRef]

14. ASPS Statement on Breast Reconstruction in the face of COVID-19 Pandemic. Available at: https://www.plasticsurgery.org/documents/medical-professionals/COVID19-Breast-ReconstructionStatement.pdf. Accessed Jun 03, 2020. 